\title{
IDENTIFICACIÓN DE LA OFERTA TURÍSTICA Y DE EXCURSIONISMO DE HERPETOFAUNA EN ECUADOR
}

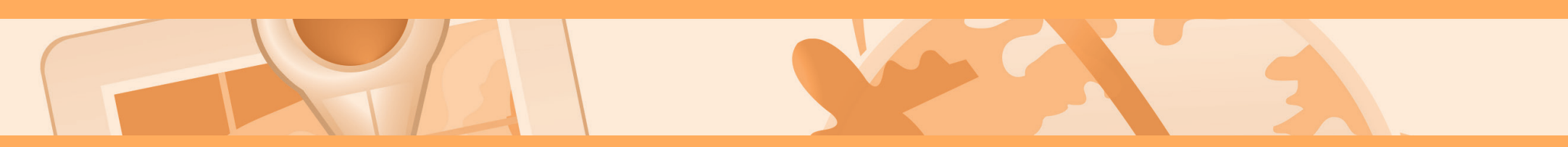

\section{Autores:}

Daniel Hernández Sánchez ${ }^{1}$

Ximena Aguirre Ulloa²

${ }^{1}$ Universidad Tecnológica Equinoccial

Investigador Asociado Facultad de Turismo, Hotelería y Gastronomía

Email: dahs37@gmail.com

Teléf.: 0984044641

${ }^{2}$ Universidad Tecnológica Equinoccial

Facultad de Turismo, Hotelería y Gastronomía 


\section{Resumen}

Se realizó un estudio de la oferta turística y de excursionismo de herpetofauna en Ecuador para establecer una línea base de establecimientos, instituciones y áreas que ofertan actividades turísticas vinculadas a la observación de anfibios o reptiles.

En este estudio se aplicaron criterios como: distribución de anfibios y reptiles en las regiones naturales del país, observación de herpetofauna en estado silvestre en sitios de alojamiento, áreas protegidas y bosques protectores, e identificación de instituciones especializadas en este grupo (oferta turística); búsqueda de instituciones de exposición y/o investigación de este grupo (oferta de excursionismo). Los resultados arrojaron información de 34 áreas protegidas con potencialidad para la observación turística de herpetofauna, de las cuales únicamente cuatro ofrecen esta actividad; en bosques protectores solo en Mindo-Nambillo se realiza actividades de este tipo.

Se determinó también que en 87 de 271 establecimientos de alojamiento se ejecutan actividades de observación de fauna en general, y en 30 , se oferta avistamiento de anfibios y reptiles. Se identificaron dos instituciones en el país que ofrecen observación de estos vertebrados como actividad principal.

En lo referente a la oferta de excursionismo se determinó que 13 de 17 museos de ciencias naturales a nivel nacional, ofrecen al público exhibiciones de especímenes de fauna en general, entre ellos muestras de anfibios y reptiles; así también se identificaron 3 de 42 Unidades de Manejo de Vida Silvestre (zoológicos y centros de rescate) que ofertan visitas guiadas y autoguiadas para observación de este grupo faunístico.

En cuanto a la observación turística de herpetofauna se estableció que existen cuatro formas de efectuar la actividad: concierto y observación de ranas, observación de caimanes, observación de cocodrilos y observación de herpetofauna en general; mientras que en el excursionismo la actividad se restringe a la observación de herpetofauna en cautiverio.

Palabras clave: herpetofauna, turismo de naturaleza, oferta turística, oferta de excursionismo 


\section{Abstract}

In the article, has been determinated the tourist offer of herpetofauna in Ecuador, and with this, the base line of touristic services, institutions and areas that offer tours according to anphibians and reptiles observation.

In this research, some of the guidelines were: distribution of amphibians and reptiles in the natural regions of the country, herpetofauna wildlife observation in lodges, national parks and private protected areas, the identification of NGO specialized in this matter (more than one day tours offered) and exposition and research centers (one day tours).

Some of the conclusions were: of 34 national parks with this potential, only four offer tours, and in private protected areas only one does: Mindo Nambillo. In 87 of 271 lodges, there is wildlife observation programs, in 30 of them, there is the chance to follow their lifestyles and habits, and in two places, these are the principal activities that tours offer.

About one day tours in this area, 13 of the 17 natural science museums around the country have a permanent fauna exhibition, including reptiles and amphibians; tree of 42 Wildlife Management Office (zoos and animals recue centers) were located, and they also offer guided and self-guiding tours for the group in concern.

About herpetofauna tour observation, there are four ways to do this activity: concert and observation of frogs, caiman observation, crocodrile observation and general herpetofauna observation, meanwhile in one day tours only herpetofauna observation un captivity is possible.

Key words: herpetofauna, nature tourism, tourist offer, one day tour offer 


\section{Introducción}

El sector turístico en Ecuador se presenta como una industria de importancia en la balanza de pagos del país, es así como en 2006 (registro más antiguo disponible) el ingreso generado por el turismo (492 millones de dólares) se posiciona en séptimo lugar dentro de las exportaciones del país; posteriormente en 2007 y 2008 el turismo se ubica en sexto lugar con 626,2 y 745,2 millones de dólares respectivamente (MINTUR, 2011, 2012).

Continuando con su tendencia positiva en 2009 y 2010 con 674 y 786,5 millones para cada año, el turismo llega al cuarto lugar, siendo estos los mejores años para el sector; tras lo cual baja al sexto lugar con 849,7 millones (2011) y en 2012 conserva dicha posición con 1032,7 millones, (MINTUR, 2012, 2013a, 2013b).

Es así como se puede afirmar que si bien el sector turístico ha variado su contribución con la balanza de pagos del país, en los últimos años este ha mantenido su importancia para la generación de divisas en la economía ecuatoriana.

Dentro de las modalidades de turismo que se practican, el turismo de naturaleza se caracteriza porque el turista o excursionista elige un destino con un fuerte componente natural, aunque su motivación no esté relacionada exclusivamente con una absoluta implicación con los valores naturales del entorno, sino más bien con el disfrute de los recursos que este ofrece; por tanto quien gusta del turismo de naturaleza pretende estar en contacto con el entorno natural aunque según el caso, con diferente grado de implicación en la misma (Flores, 2007).

Conociendo que el turismo de naturaleza es una modalidad turística que incluye entre sus actividades la observación de flora y fauna, se puede indicar que las preferencias de actividades de turistas nacionales reflejan que $26,1 \%$ disfruta visitar naturaleza o áreas protegidas, y más específicamente $3 \%$ prefiere realizar actividades de observación de flora y fauna (MINTUR, 2006).
Paralelamente Ecuador ocupa el séptimo lugar entre los 17 países megadiversos del mundo y el primer lugar si se considera su número de especies de vertebrados por unidad de área (Coloma, 2001). En cuanto a la diversidad de anfibios, en la actualidad se estima que existen 7.192 especies formalmente descritas (Frost, 2014); Ecuador ocupa el tercer lugar en el mundo en cuanto a diversidad de anfibios con 544 especies registradas (hasta diciembre 2013), conformadas por los órdenes: Anura, Caudata, y Gymnophiona; lo que constituye el $7,6 \%$ del total de especies de anfibios a nivel mundial, en un territorio de 256.370 km2 (Ron, S. R., Guayasamin, J. M. Yanez-Muñoz, M. H. \&Merino-Viteri, A., 2013).

El grupo de los reptiles en el país está constituido por tres órdenes: Chelonia, Crocodylia y Squamata. Éstos llegan a 431 especies registradas (hasta diciembre 2013), representando un 4,4\% de las 9.766 especies registradas a nivel mundial (Uetz, 2013); gracias a esto el país ocupa el décimo lugar en número de especies de reptiles en el mundo, en un territorio que ocupa apenas el 0,05\% de la superficie mundial (Torres-Carvajal, O., D. Salazar-Valenzuela \& A. Merino-Viteri, 2013).

Vinculando el desarrollo del sector turístico y la diversidad herpetofaunística en el país, se han manifestado nuevas actividades dentro del turismo de naturaleza, mismas que se caracterizan y motivan por la observación de herpetofauna; no obstante ante la carencia de un estudio formal acerca de dichas actividades en el país, se planteó en el año 2012 el estudio titulado "Análisis de la oferta turística y de excursionismo de herpetofauna en Ecuador", estableciendo ofertantes y actividades a fin de conocer la actual situación sobre la utilización turística de anfibios y reptiles en el país.

\section{Método y Materiales}

Para identificar la oferta objeto de estudio se decidió omitir el cálculo de una muestra, realizando en su lugar, el análisis de la totalidad del universo, el cual que obtenido en base a los catastros y registros del Ministerio del Ambiente (áreas protegidas, bosques 
protectores y unidades de manejo de vida silvestre), Ministerio de Turismo (establecimientos de alojamiento) y Ministerio de Cultura (museos); además de la revisión bibliográfica del Plan estratégico de desarrollo de turismo sostenible para Ecuador (PLANDETUR).

Finalmente los registros obtenidos fueron discriminados en base a criterios lógicos de búsqueda y posteriormente analizados mediante encuestas estructuras, mismas que fueron aplicadas a cada uno de los establecimientos a fin de identificar la oferta en cuestión.

\section{Desarrollo Temático}

El estudio inició con la diferenciación entre oferta turística y oferta de excursionismo para la observación de herpetofauna, en relación a los conceptos de turista y excursionista mencionados en Montaner et al., (1998).

Tomando en cuenta estos conceptos, se puede afirmar que en la oferta turística, el turista permanece más de 24 horas realizando al menos una pernoctación y en la de excursionismo el visitante permanece menos de 24 horas, sin realizar pernoctación alguna; en ambos casos motivados principalmente por la observación de anfibios y/o reptiles.

\section{Oferta turística de herpetofauna}

A fin de que se realice la pernoctación, el turista necesita encontrarse en un sitio de alojamiento durante su visita, el que a su vez deberá estar rodeado de espacios naturales, para hacer posible la observación de herpetofauna en estado silvestre.

Es así que la búsqueda de la oferta turística se inició con la identificación de la distribución de anfibios y reptiles en el país, con base en base Ron et al. (2013) y Torres-Carvajal et al. (2013), con la finalidad de conocer las regiones con mayor diversidad herpetofaunística, dentro de las cuales se ubicarían los sitios de alojamiento que oferten actividades de observación de estos vertebrados en estado silvestre.
Como se señala en la Tabla 1 se identificó siete regiones naturales con riqueza en herpetofauna, mientras que tres regiones constan como regiones de baja diversidad.

Tabla 1. Relación entre regiones naturales y diversidad de herpetofauna

\begin{tabular}{|c|c|c|}
\hline ANFIBIOS & REGIONES NATURALES PARA HERPETOFAU & REPTILES \\
\hline \multirow[t]{2}{*}{$\begin{array}{l}\text { species } \\
\text { umerosas }\end{array}$} & $\begin{array}{l}\text { Bosque montano oriental } \\
\text { Bosque montano occidental } \\
\text { Bosque piemontano occidental } \\
\text { Bosque piemontano oriental }\end{array}$ & $\begin{array}{l}\text { Especies } \\
\text { escasas }\end{array}$ \\
\hline & $\begin{array}{l}\text { Bosque húmedo tropical amazónico } \\
\text { Bosque húmedo tropical del choco }\end{array}$ & \multirow{2}{*}{$\begin{array}{l}\text { Especies } \\
\text { numerosas }\end{array}$} \\
\hline \multirow{2}{*}{ species escasas } & $\begin{array}{c}\text { Bosque deciduo de la costa (Región Tumbesina) } \\
\text { Galápagos }\end{array}$ & \\
\hline & $\begin{array}{l}\text { Páramo } \\
\text { Matorral interandino }\end{array}$ & $\begin{array}{l}\text { Especies } \\
\text { escasas }\end{array}$ \\
\hline
\end{tabular}

Una vez identificadas las regiones de alta diversidad en herpetofauna, se analizó los lugares ideales para realizar observación, señalados en el PLANDETUR, discriminando los sitios de acuerdo a su pertinencia con las regiones antes identificadas.

Resultado de este análisis, fueron establecidos 16 lugares para observación de fauna que ingresaron en la búsqueda de la oferta para luego ser clasificados y seleccionados según sus características en tres categorías sobre las cuales se desarrollaría la búsqueda de establecimientos de alojamiento turístico.

Tabla 2.Productos de ecoturismo del PLANDETUR y categorización

\section{PRODUCTOS}

CATEGORÍAS

\section{Islas Galápagos}

Parque Nacional Yasuní

Reserva de Producción Faunística Cuyabeno

Parque Nacional Sangay

La Ruta del Sol: Parque Nacional Machalilla

Parque Nacional Podocarpus

Reserva Ecológica Cotacachi-Cayapas

Reserva Ecológica de Limoncocha

Reserva Ecológica Cayambe-Coca

Bosque Petrificado de Puyango

Bosque Nublado de Mindo Nambillo

Napo Wildlife Center

Reserva Ecológica Kapawi

La Ruta del Sol: Puerto López

Lodges Amazónicos

La Ruta de Orellana
Áreas protegidas

\begin{tabular}{|l|l|} 
& \\
\hline Bosques protectores \\
\hline Establecimientos de alojamiento \\
turístico
\end{tabular}




\section{Áreas protegidas}

Todas las áreas protegidas del país se encuentran incluidas dentro del Sistema Nacional de Áreas Protegidas (SNAP), conformado por cuatro subsistemas: Subsistema Patrimonio de Áreas Naturales del Estado (PANE), Subsistema de Gobiernos Autónomos Descentralizados, Subsistema de Áreas Protegidas Comunitarias y Subsistema de Áreas Protegidas Privadas; de los cuales tan solo el PANE se encuentra organizado en su totalidad, mientras que los otros tres restantes se encuentran en proceso de organización (Ministerio del Ambiente, 2009)

EI PANE cuenta con 47 áreas protegidas, mismas que fueron analizadas según la distribución de herpetofauna en el país, logrando identificar 34 áreas dentro de las regiones de alta diversidad herpetofaunística y 13 áreas en las regiones de baja diversidad.

Tras identificar las áreas de alta diversidad de anfibios y/o reptiles y considerando que el Ministerio del Ambiente (MAE) se encarga de controlar la operación de las empresas turísticas en las áreas protegidas del estado, se aplicó una encuesta estructurada a los jefes de áreas, a fin de conocer la utilización o no de herpetofauna en actividades turísticas y las empresas de alojamiento vinculadas con las áreas protegidas.

\section{Bosques Protectores}

Los bosques protectores representan otro tipo de espacios naturales donde se puede llevar a cabo la observación turística de herpetofauna, no obstante en su actual situación existen varios propietarios para un mismo bosque (comunidades campesinas e indígenas, fundaciones, municipios e incluso propietarios individuales), por lo que no se dispone de un registro de los propietarios de éstos a nivel nacional, y el contacto con los mismos es prácticamente imposible.

Con este antecedente se identificó la oferta dentro de los bosques señalados como productos para ecoturismo dentro del PLANDETUR; estableciendo contacto para determinar las actividades que se realizan en el Bosque Petrificado de Puyango, y registrando las actividades de los sitios de alojamiento del Bosque Nublado de Mindo Nambillo.

\section{Galápagos}

El Archipiélago de Galápagos es una región de considerable diversidad de reptiles endémicos en casi la totalidad de las islas, mientras que los anfibios se limitan a ciertas especies invasoras, esto sumado a la presencia de las áreas protegidas Parque Nacional Galápagos y Reserva Marina Galápagos, constituyen el producto estrella del ecoturismo en el país, por lo cual Galápagos fue analizado con base a las actividades registradas por el MAE en sus dos áreas protegidas y los establecimientos de alojamiento turístico (cabañas y hosterías) existentes en el lugar.

\section{Establecimientos de alojamiento turístico}

Para la búsqueda de sitios de alojamiento se analizaron hosterías y cabañas registradas por el MINTUR en el catastro de establecimientos turísticos, ya que son los únicos establecimientos que pueden encontrarse relacionados con sitios naturales (MINTUR, 2002).

Los establecimientos fueron analizados dentro de la categoría de lujo, primera y segunda clase; excluyendo a las categorías tercera y cuarta debido a la confiabilidad de la información disponible, así también se utilizó la distribución de herpetofauna para establecer los establecimientos que se encontraban en zonas de alta diversidad.

Finalmente se identificaron 312 establecimientos de alojamiento turístico, los cuales junto con los productos para ecoturismo del PLANDETUR (2) y los sitios de alojamiento del PANE (21) y Galápagos (9) que no constaban en el catastro, sumaron un total de 342 establecimientos para el país.

Determinado el número de establecimientos, se contactó al $79 \%$ de éstos (271), a fin de conocer las actividades que ofertan en relación con la herpetofauna, mediante la aplicación de una encuesta, logrando así establecer la oferta turística de acuerdo a las actividades ejecutadas en estos establecimientos. 
Identificación de la oferta turística y de excursionismo de herpetofauna en Ecuador

\section{Instituciones especializadas}

Paralelamente se identificaron instituciones privadas que utilizan anfibios y/o reptiles en actividades turísticas, para lo cual se implementaron encuestas en centros de investigación de herpetofauna existentes en las universidades del país, además de los identificados durante la búsqueda de la oferta de excursionismo. Posteriormente se realizaron entrevistas a los directivos de éstas para corroborar la información obtenida.

\section{Oferta de excursionismo de herpetofauna}

Considerando que la oferta de excursionismo buscada se caracteriza por permitir la observación de herpetofauna durante una visita de menos de 24 horas y sin pernoctación alguna, los museos de ciencias naturales y los zoológicos son los únicos sitios que cumplen con dichas particularidades, razón por la cual conformarán las dos categorías sobre las cuales basar la búsqueda de la oferta en cuestión.

A diferencia de la oferta de turismo, en la oferta de excursionismo la distribución de herpetofauna es irrelevante, debido a que los especímenes vivos permanecen en cautiverio (zoológicos) o en su defecto se trata de especímenes conservados (museos).

\section{Museos de Ciencias Naturales}

Haciendo relación al catastro de museos del Sistema Ecuatoriano de Museos (SIEM) del Ministerio de Cultura de Ecuador, se identificaron 17 museos de ciencias naturales en el país, los que fueron analizados de acuerdo a la exhibición de muestras herpetológicas, a fin de conocer su especialización o no sobre un grupo en especial, lo que repercute de manera directa en la motivación de los visitantes que acuden al museo.

\section{Unidades de Manejo de Vida Silvestre}

De forma similar al análisis de museos, las UMVS (zoológicos y centros de rescate) fueron analizadas con base al registro del Ministerio del Ambiente de Ecuador, identificándose un total de 42 sitios que alojan anfibios o reptiles vivos en el país.
Se implementó también un análisis de estos centros tomando en cuenta el número de especímenes de herpetofauna que éstos albergan, para determinar su especialización sobre algún grupo faunístico.

\section{Resultados}

\section{Oferta turística}

Tras aplicar las encuestas a los jefes de área de las 34 áreas protegidas dentro de regiones de alta diversidad herpetológica, se determinó que dentro de 4 áreas se realizan actividades turísticas vinculadas con la herpetofauna, éstas son: Parque Nacional Yasuní, Reserva Biológica Limoncocha, Reserva de Producción Faunística Cuyabeno y Área Nacional de Recreación Isla Santay y Gallo, que representan el $12 \%$ del total de áreas protegidas en regiones de alta diversidad en herpetofauna. En cuanto a los bosques protectores analizados, el bosque de Puyango no registró ninguna actividad turística vinculada con la herpetofauna, mientras que Mindo Nambillo fue analizado a nivel de sitios de alojamiento turístico. De los 271 establecimientos de alojamiento contactados, en el 32\% se realiza actividades de observación de fauna (87 establecimientos) mientras que el $68 \%$ restante no utiliza fauna en sus actividades ofertadas.

Así también dentro de los 87 establecimientos identificados, en el $66 \%$ (57) se realizan actividades de observación de fauna no motivadas exclusivamente por la observación de herpetofauna, sino mas bien por la diversidad faunística en general existente en el sitio o se especializan en otro grupo de animales; por su parte el 34\% restante (30 establecimientos) ofertan actividades cuya motivación principal es la observación de anfibios y/o reptiles. Los 30 establecimientos de alojamiento identificados forman parte de la oferta turística de herpetofauna, así también dentro de estos centros se realizan tres actividades vinculadas con el uso de anfibios y/o reptiles las cuales son: observación de caimanes, observación de cocodrilos y concierto y observación de ranas. En cuanto a las instituciones especializadas se encontró dos instituciones que se enfocan en la utilización turística de herpetofauna en general, éstas son Tropical Herping y Wikiri, 
no obstante al momento tan solo Tropical Herping se encuentra ofreciendo la actividad, mientras que Wikiri planea implementarla en un futuro próximo.

En la Tabla 3 se puede observar la oferta turística de herpetofauna identificada en el país, junto con las actividades que se realizan y la zona donde operan.

Tabla 3. Oferta turística de herpetofauna identificada

\begin{tabular}{|c|c|c|}
\hline OFERTA & UBICACIÓN & ACTIVIDAD \\
\hline $\begin{array}{l}\text { Cabañas Limoncocha } \\
\text { Pusara } \\
\text { Cabañas Supay Cocha }\end{array}$ & $\begin{array}{l}\text { Reserva Biológica } \\
\text { Limoncocha }\end{array}$ & \multirow{6}{*}{ Observación de caimanes } \\
\hline $\begin{array}{l}\text { Sacha Lodge } \\
\text { Yuturi Lodge } \\
\text { Napo Wildlife Center } \\
\text { Yarina Ecolodge } \\
\text { La Selva Jungle Lodge } \\
\text { Manatee Amazon Explorer } \\
\text { Shiripuno Lodge } \\
\text { Huaorani Ecolodge } \\
\text { Sani Lodge }\end{array}$ & Parque Nacional Yasuní & \\
\hline $\begin{array}{l}\text { Cuyabeno Lodge } \\
\text { Cuyabeno River Lodge } \\
\text { San Francisco Lodge } \\
\text { Jamu Lodge } \\
\text { Samona Lodge } \\
\text { Hormiga Lodge } \\
\text { Hot Spots Lodge } \\
\text { Siona Lodge } \\
\text { Magic River Lodge } \\
\text { Guacamayo Ecolodge } \\
\text { Tapir Lodge } \\
\text { Amaru Lodge } \\
\text { Cayman Lodge }\end{array}$ & $\begin{array}{l}\text { Reserva de Producción } \\
\text { Faunística Cuyabeno }\end{array}$ & \\
\hline $\begin{array}{l}\text { Kapawi Ecolodge } \\
\text { Yacuma Lodge }\end{array}$ & Reserva Privada & \\
\hline Flotel Misión Orellana & Río Napo & \\
\hline Flotel Jungle Discovery & Río Cuyabeno & \\
\hline $\begin{array}{l}\text { Tropical Herping } \\
\text { Wikiri }\end{array}$ & Varias reservas privadas & $\begin{array}{l}\text { Observación de } \\
\text { herpetofauna en general }\end{array}$ \\
\hline Las Palmeras de Santay & $\begin{array}{l}\text { Área Nacional de } \\
\text { Recreación Isla Santay } \\
\text { y Gallo }\end{array}$ & Observación de cocodrilos \\
\hline Mindo Lago & $\begin{array}{l}\text { Bosque Nublado Mindo } \\
\text { Nambillo }\end{array}$ & ranas \\
\hline
\end{tabular}

Observación de Herpetofauna en general. Se basa en la búsqueda de cualquier tipo de anfibio y/o reptil, por lo que la actividad está motivada exclusivamente por la búsqueda y observación de herpetofauna; esta actividad constituye la oferta turística de herpetofauna propiamente dicha.

Concierto y observación de ranas. La actividad se enfoca al disfrute de los cantos de anuros machos en un determinado lugar, tras lo cual se continúa con la búsqueda de los mismos hasta localizarlos visualmente.

Observación de cocodrilos. Es una actividad realizada exclusivamente en el Área Nacional de Recreación Isla Santay y Gallo, se basa en realizar un recorrido por un sendero que concluye en una piscina de reproducción de cocodrilos donde se puede observar a los especímenes en forma directa.

Observación de caimanes. La actividad se desarrolla mediante recorridos nocturnos en canoa, durante los cuales se avista a los especímenes en sus lugares de hábitat; se trata de la actividad más realizada por los establecimientos de alojamiento y se concentra en tres áreas protegidas del PANE nombradas con anterioridad.

\section{Oferta de excursionismo}

Una vez analizadas las muestras expuestas en los 17 museos de ciencias naturales del país se identificaron 13 museos (76\%) no especializados en la exhibición específica de un grupo de animales, sino que ofrecen exhibición de fauna general sin que se dé énfasis a un grupo en particular. En consecuencia la motivación de la visita a estos sitios no recae específicamente sobre la observación de herpetofauna, sino mas bien sobre toda la muestra del museo.

\section{Un 24\% restante (4 museos) ofrecen una muestra especializada} en algún tipo de fauna, es así como se identifican tres muesos enfocados en megafauna y uno en cetáceos, por lo cual la visita a dichos lugares estará motivada por la observación de dichos grupos. 
Identificación de la oferta turística y de excursionismo de herpetofauna en Ecuador

No obstante ningún museo natural del país está enfocado en herpetofauna, por lo que no existe oferta de excursionismo dentro de esta categoría.

Por otra parte, las 42 UMVS fueron analizadas de acuerdo al porcentaje de herpetofauna en relación al total de individuos alojados en éstas, dando como resultado que en el 83\% de unidades (35) los anfibios y reptiles representan menos de la cuarta parte del total de especies alojadas, mientras que en el $10 \%$ de unidades (4) la herpetofauna representa menos de la mitad de las especies albergadas.

Finalmente en un $7 \%$ de establecimientos ( 3 UMVS) la herpetofauna representa más de la mitad de las especies alojadas, encontrándose en estas unidades la oferta de excursionismo buscada, siendo los sitios identificados: Vivarium de Quito, Bioparque Amaru de Cuenca y Serpentario de San Martin.

La oferta en Galápagos no presenta establecimiento alguno que realice actividades turísticas especializadas en herpetofauna en ninguna de las dos áreas protegidas; sin embargo se localizaron tres centros de crianza de tortugas gigantes (galapagueras), los cuales albergan ejemplares del género Chelonoidis, que son observados por los excursionistas. De acuerdo a lo mencionado los centros Fausto Llerena, Cerro Colorado y Arnaldo Tupiza se incluyen dentro de la oferta de excursionismo para herpetofauna.

Finalmentedurantelabúsqueda deinstituciones especializadas se estableció que la compañía de Biocomercio Wikiri se encuentra próxima a ofertar una actividad denominada "RanAventura", donde se realizan recorridos en senderos dentro de un ambiente controlado en las instalaciones de Wikiri, con la finalidad de observar anuros en ambientes recreados, por lo cual la actividad se enmarca dentro de la oferta de excursionismo.

En la Tabla 4 se puede observar el listado de la oferta de excursionismo de herpetofauna identificada en el país.
Tabla 4. Oferta de excursionismo de herpetofauna identificada

\begin{tabular}{|c|c|c|}
\hline OFERTA & UBICACIÓN & CATEGORÍA \\
\hline $\begin{array}{l}\text { Vivarium } \\
\text { Bioparque Amaru } \\
\text { Serpentario de San Martín }\end{array}$ & $\begin{array}{l}\text { Quito } \\
\text { Cuenca } \\
\text { Baños de Agua } \\
\text { Santa }\end{array}$ & $\begin{array}{l}\text { Unidad de Manejo de Vida } \\
\text { Silvestre }\end{array}$ \\
\hline $\begin{array}{l}\text { Centro de Crianza Fausto Llerena } \\
\text { Centro de Crianza Fausto Arnaldo } \\
\text { Tupiza } \\
\text { Galapaguera Cerro Colorado }\end{array}$ & $\begin{array}{l}\text { Galápagos } \\
\text { Galápagos } \\
\text { Galápagos }\end{array}$ & $\begin{array}{l}\text { Centro de Crianza de } \\
\text { Galápagos }\end{array}$ \\
\hline Wikiri & Quito & Ofertas futuras \\
\hline
\end{tabular}

\section{Discusión}

Los resultados obtenidos en la investigación demuestran que en el país existen cuatro áreas protegidas, 30 establecimientos de alojamiento y dos instituciones biológicas, enfocadas en el uso turístico de anfibios y reptiles; mientras que en el excursionismo se identifican siete Unidades de Manejo de Vida Silvestre (zoológicos, centros de crianza de tortugas e instituciones biológicas).

Mediante el análisis de las áreas protegidas, se determinó que la mayor parte de las mismas se encuentran en regiones de alta diversidad de herpetofauna; sin embargo, la observación turística de dicho grupo se restringe a 4 áreas del PANE. Así también los establecimientos de alojamiento son los mayores ofertantes del uso turístico de herpetofauna, debido a su ubicación y diversificación de actividades ofertadas. Por otra parte las entidades especializadas son las que mayor relación guardan con el uso turístico de herpetofauna, sin dejar de lado su enfoque biológico.

Las actividades de herpetofauna identificadas dentro de la oferta turística se caracterizan por establecer un contacto multisensorial entre el observador y el espécimen, motivando el interés de turistas.

En lo que respecta al excursionismo, los ofertantes se limitan a Unidades de Manejo de Vida Silvestre donde la única actividad existente es la observación de fauna en cautiverio. 
Con el estudio se establece la primera línea base de ofertantes de actividades turísticas con el uso de herpetofauna en el país, dando a conocer una alternativa más dentro del turismo de naturaleza.

\section{Conclusiones}

Con la identificación de la oferta de herpetofauna para turismo y excursionismo se pone de manifiesto la presencia de una nueva tendencia en las actividades del turismo de naturaleza en el país.

La mayor parte de la oferta turística se encuentra ubicada en regiones de alta diversidad de herpetofauna: Bosque Húmedo Tropical Amazónico y Bosque Húmedo Tropical del Chocó.

Las entidades especializadas que han partido desde el ámbito biológico y han incursionado en la actividad turística muestran un manejo técnico en la utilización de anfibios y reptiles, contrastando con el manejo empírico del resto de ofertantes en esta área.

Mediante el diagnóstico obtenido a partir del registro generado, se apertura la posibilidad de desarrollar investigaciones enfocadas a fortalecer el desarrollo del turismo herpetológico en el país.

\section{Referencias Bibliográficas}

Coloma, L. \& Ron, S. (2001). Ecuador megadiverso: anfibios, reptiles, aves, mamíferos. Quito: Museo de Zoología, Centro de Biodiversidad y Ambiente, Departamento de Ciencias Biológicas, Pontificia Universidad Católica del Ecuador.

Flores, R. (2007). Competitividad sostenible de los espacios naturales protegidos como destinos turísticos: Un análisis comparativo de los parques naturales Sierra de Aracena y Picos de Aroche y Sierras de Cazorla, segura y las villas.(Tesis doctoral). Universidad de Huelva, Huelva

Frost, Darrel R. 2014. Amphibian Species of the World: an Online Reference. Version 6.0 (Date of access).

Electronic Database accessible at http://research.amnh.org/ herpetology/amphibia/index.html. American Museum of Natural History, New York, USA.
Ministerio de Turismo (2013a). La experiencia turística en el Ecuador: Cifras esenciales del turismo interno y receptor. Quito: MINTUR

Ministerio de Turismo (2013b). Publicaciones Agosto 2013. Quito: MINTUR

Ministerio de Turismo (2012). Publicaciones Diciembre 2012. Quito: MINTUR

Ministerio de Turismo (2011). Boletín de estadísticas turísticas 2006 2010. Quito: MINTUR

Ministerio de Turismo. (2006). Plan estratégico de desarrollo de turismo sostenible para Ecuador. Quito: MINTUR

Ecuador. Ministerio de Turismo. (2002). Reglamento general de actividades turísticas. Quito: MINTUR

Ministerio del Ambiente del Ecuador (2009). Incorporación de Subsistemas de Áreas Protegidas Privadas, Comunitarias, Indígenas y Afroecuatorianas y de Gobiernos Seccionales al Sistema Nacional de Áreas Protegidas. Informe Final de Consultoría. Fabara\& Compañía Abogados. Programa GESOREN-GTZ. Quito.

Montaner, J., Antich, J. \&Arcarons, R. (1998). Diccionario de turismo. Madrid: Síntesis

Ron, S. R., Guayasamin, J. M., Yanez-Muñoz, M. H., Merino-Viteri, A. y Ortiz, D. A. 2013. AmphibiaWebEcuador. Version 2013.0. Museo de Zoología, Pontificia Universidad Católica del Ecuador. < http://zoologia.puce.edu.ec/Vertebrados/anfibios/ AnfibiosEcuador>, acceso 15 de diciembre, 2013

Torres-Carvajal, O., D. Salazar-Valenzuela y A. Merino-Viteri. 2013. ReptiliaWebEcuador. Versión 2014.0. Museo de Zoología QCAZ, Pontificia Universidad Católica del Ecuador. <http:// zoologia.puce.edu.ec/Vertebrados/reptiles/reptilesEcuador>, acceso 15 de diciembre, 2013

Uetz, P. \&JiríHošek (eds.), TheReptileDatabase, http://www.reptiledatabase.org, acceso 15 de diciembre, 2013 